\title{
Effect of oral nifedipine on ocular blood flow in patients with low tension glaucoma
}

\author{
Orna Geyer, Meira Neudorfer, Ada Kessler, Edit Firsteter, Moshe Lazar, Yhoshua Almog
}

\begin{abstract}
Aim-To investigate the effect of oral nifedipine on ocular blood flow in patients with low tension glaucoma (LTG).

Methods-In this prospective study we examined the effects of 3 weeks of treatment with oral nifedipine $30 \mathrm{mg} / \mathrm{day}$ in 11 patients with LTG, by using colour Doppler ultrasound imaging to measure haemodynamic variables in the central retinal (CRA), short posterior ciliary (SPCA), and ophthalmic (OA) arteries. Intraocular pressure (IOP) and blood pressures were also evaluated.
\end{abstract}

Results-Nifedipine failed to alter IOP nor did it change peak systolic velocity, end diastolic velocity, or the resistance index in any of the three ocular vessels studied ( $p$ >0.05). However systolic and diastolic systemic arterial blood pressure measurements varied significantly after nifedipine treatment compared with baseline $(\mathbf{p}<0.05)$.

Conclusion-Our study failed to demonstrate a significant effect of nifedipine on retrobulbar circulation of patients with LTG.

(Br F Ophthalmol 1996;80:1060-1062)

Vasospasm has been suggested to play a role in the pathogenesis of low tension glaucoma (LTG). ${ }^{1}$ Several recent reports demonstrated that patients with LTG have a reduced blood velocity in the ophthalmic ${ }^{2}$ and central retinal arteries compared with normal controls. ${ }^{3}$ Because calcium channel blockers reduce vascular resistance, ${ }^{4}$ they were evaluated for potential benefit in patients with LTG ${ }^{5-8}$

Colour Doppler imaging (CDI) is a noninvasive method for quantifying blood flow of the retrobulbar circulation in vivo. This ultrasound technique combines B-scan grey scale imaging of tissue structure, coloured representation of blood flow based on Doppler shifted frequencies and pulsed Doppler measurement of blood flow velocities.

We used CDI to study the effects of a systemic calcium channels blocker, nifedipine, on ocular circulation of patients with LTG.

\section{Methods}

Eleven patients (seven women and four men, mean age 71.6 (SD 5.5) years) participated in the study. All patients had bilateral LTG as diagnosed by the presence of a visual field defect and glaucomatous optic disc cupping in addition to an intraocular pressure (IOP) of less than $20 \mathrm{~mm} \mathrm{Hg}$ (no medication) with nor- mal diurnal curve measurements. The anterior chamber angle was open on gonioscopy. A computed tomography scan of the head, carotid ultrasonography, complete blood cell count, evaluation for syphilis titres, Westergren erythrocyte sedimentation rate, and antinuclear antibody titres were all within normal limits. The study was approved by the institutional review board, and informed consent was obtained from all patients. Patients stopped using antiglaucoma drugs 3 weeks before entering the study. Baseline IOP, systemic blood pressure, and colour Doppler ultrasound values were measured. After the initial visit, patients were treated with nifedipine $10 \mathrm{mg}$ three times daily for 3 weeks. At the completion of the treatment period, the three measurements were repeated.

IOP was determined by Goldmann applanation tonometry, and arterial blood pressure was measured by sphygmomanometry. Sonographic examination was performed using a colour Doppler (Acuson128XP10 Mountain View, CA, USA) with a $7.5 \mathrm{MHz}$ linear transducer. Both eyes of each patient were examined according to the protocol described previously by Lieb et al. ${ }^{9}$ Scanning was performed in the axial view, thereby obtaining an overall view and permitting the identification of the precise location and direction of flow in the vessels. Vascular flow was evaluated by both colour and spectral Doppler modes. The Doppler angle was kept below 60 degrees. Blood flow measurements were studied for the ophthalmic (OA), short posterior ciliary (SPCA), and central retinal (CRA) arteries. CRA measurements were performed at approximately $2 \mathrm{~mm}$ posterior to the globe.

Posterior ciliary vessels were located either temporally or nasally adjacent to the optic nerve shadow. The same site was studied for subsequent measurements in each subject. The peak systolic, end diastolic, and the resistance index (RI) were calculated for each of the vessels tested. PSV refers to the highest blood flow velocity achieved during systole and is calculated from the frequency of the peak in the Doppler shift spectral waveform. EDV refers to the lowest velocity occurring during diastole and is calculated from the frequency of the trough in the waveform. $\mathrm{RI}$ is defined as the difference between the maximum and minimum velocities of one cardiac cycle divided by the maximum velocity ( $R I=P S V-E D V / P S V)$ and is a measure of peripheral vascular resistance.

The paired two tailed $t$ test was used to evaluate the statistical significance of change from baseline of each of the measured 
Table 1 Blood pressure and intraocular pressure before and after treatment with nifedipine (mean (SEM)) in patients with low tension glaucoma

\begin{tabular}{lccc}
\hline & \multicolumn{2}{c}{ Nifedipine } & \\
\cline { 2 - 3 } & Baseline & Drug & \\
\hline Blood pressure (mm Hg) & $147(9)$ & $136(6.7)$ & $0.04^{\star}$ \\
$\begin{array}{c}\text { Systolic } \\
\text { Diastolic }\end{array}$ & $87(6)$ & $78(3)$ & $0.03^{\star}$ \\
$\begin{array}{l}\text { Intraocular pressure } \\
\text { (mm Hg) }\end{array}$ & $15(1)$ & $16(2)$ & $\mathrm{NS} \dagger$ \\
\hline
\end{tabular}

*Statistically significant.

†NS=not significant.

variables. A p value of less than. 0.05 was considered significant. One eye from each patient was used randomly for statistical analysis.

\section{RESULTS}

In this prospective study, no statistically significant difference in IOPs was noted after 3 weeks of treatment with nifedipine $30 \mathrm{mg} /$ day compared with baseline values $(p>0.05$, Table 1). However, systolic and diastolic blood pressure measurements after nifedipine treatment varied significantly compared with those at baseline $(p<0.05$, Table 1$)$.
A

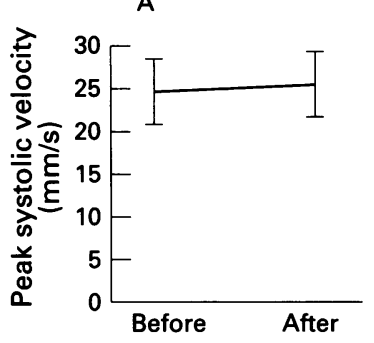

B

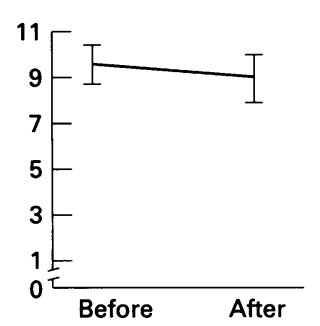

C

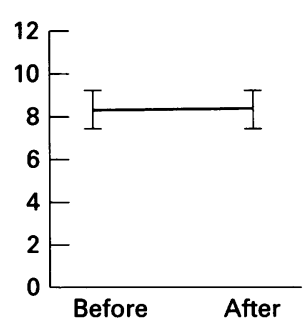

Figure 1 Colour Doppler ultrasound measurements of peak systolic velocity at baseline and after 3 weeks of treatment with nifedipine $30 \mathrm{mg} /$ day in the ophthalmic artery $(A)$, posterior ciliary (B), and central retinal (C) arteries. Nifedipine did not change this velocity in any vessel. Eleven patients with low tension glaucoma were tested. Values represent mean (SEM).
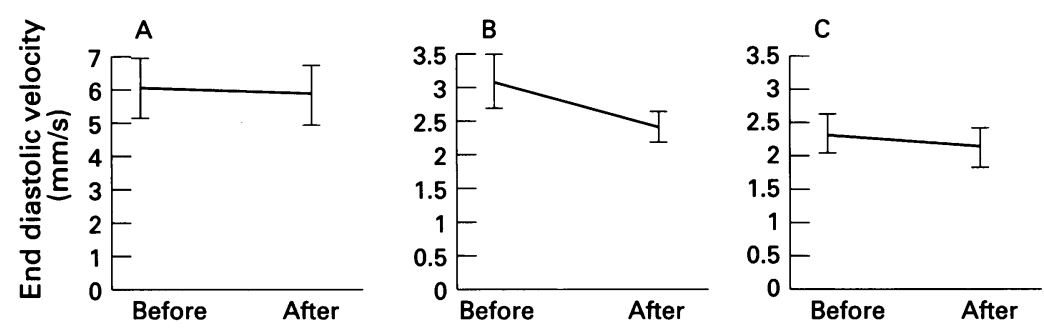

Figure 2 Colour Doppler ultrasound measurements of end diastolic velocity at baseline and after 3 weeks of treatment with nifedipine $30 \mathrm{mg} /$ day in the ophthalmic $(A)$,posterior ciliary $(B)$, and central retinal $(C)$ arteries. Nifedipine did not change this velocity in any vessel. Eleven patients with low tension glaucoma were tested. Values represent mean (SEM).
A

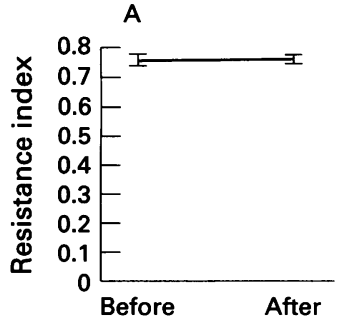

B

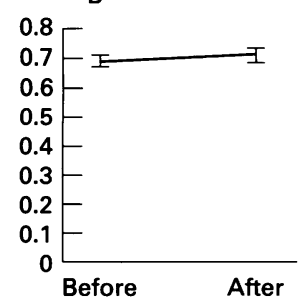

C

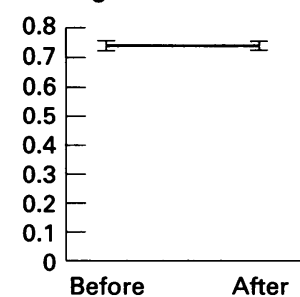

Figure 3 Colour Doppler ultrasound measurements of the resistance index at baseline and after 3 weeks of treatment with nifedipine $30 \mathrm{mg} /$ day, in the ophthalmic $(A)$, posterior ciliary $(B)$, and central retinal $(C)$ arteries. Nifedipine did not change the resistance index in any vessel. Eleven patients with low tension glaucoma were tested. Values represent mean (SEM).
Colour Doppler measurements were performed in the OA, CRA, and SPCA. Nifedipine did not significantly alter PSV, EDV, and the $\mathrm{RI}$ in any of the three ocular vessels studied ( $p>0.05$, Figs 1,2 , and 3 ).

\section{Discussion}

Most studies agreed that calcium channel blockers have a beneficial effect on visual functions of patients with LTG. Gasser and Flammer ${ }^{5}$ and Kitazawa et $a l^{6}$ reported that visual fields with various scotomata of some vasospastic LTG patients improved after the administration of calcium channels blockers. In a retrospective review of the charts of patients with normal tension glaucoma Netland et al observed that those on calcium channel blockers (diltiazem, nifedipine, and verapamil) appeared to do better than those not on the drugs, ${ }^{7}$ while nimodipine improved contrast sensitivity in LTG patients. ${ }^{8}$

It is believed that calcium channel antagonists influence visual function in patients with LTG by improving blood supply to the optic nerve head. We evaluated the effect of oral nifedipine on ocular haemodynamics using colour Doppler ultrasound imaging. Our measurements of the OA, SPCA, and CRA showed no significant differences in PSV, EDV, or RI results compared with baseline values after treatment with nifedipine. These findings indicate that oral nifedipine does not affect ocular vascular resistance in patients with LTG. The same results were reported by Evans et $a l^{10}$ who found that a 1 month treatment with oral nifedipine $(60 \mathrm{mg} /$ day $)$ did not change the results of the three variables in the same three arteries. In addition, in that study nifedipine treatment failed to alter IOP or arterial blood pressure, nor did it affect visual function or contrast sensitivity. The findings of our study differ in that we documented a drop in the systemic arterial blood pressure. Although systemic calcium channels blockers do not seem to influence ocular vascular resistance in patients with LTG topical verapamil did reduce vascular resistance in the CRA of normal volunteers. ${ }^{11}$

Colour Doppler imaging was applied for imaging and measurement of blood flow velocities in orbital vessels in a number of investigational studies and was shown to offer reproducible measures in the ophthalmic and central retinal arteries. Measures in the SPCAs are variable and thus less reliable. ${ }^{12}$ Therefore, the lack of change in the blood flow velocities after treatment with nifedipine in the ophthalmic and central retinal arteries in our patients with LTG may be of clinical significance. Limitations of this study include a relatively small number of subjects and no control group for comparison. Nevertheless, our study shows that oral nifedipine does not alter ocular blood flow velocity and vascular resistance in patients with LTG. Its significance remains to be determined.

1 Carter CJ, Brooks DE, Doyle DL, Drance SM. Investigations into a vascular etiology for low-tension glaucoma. tions into a vascular etiology

2 Harris A, Sergott RC, Speath GL, Katz JL, Shoemaker JA, Martin BJ. Color Doppler analysis of ocular vessel blood 
velocity in normal-tension glaucoma. Am $f$ Ophthalmol 1994;118:642-9.

3 Butt Z, McKillop G, O'Brien C, Allan P, Aspinall P Measurement of ocular blood flow velocity using color Doppler imaging in low tension glaucoma. Eye 1995;9:2933.

4 Braunwald E. Mechanism of action of calcium-channel blocking agents. $N$ Engl $₹$ Med 1982;307:1618-27.

Gasser P, Flammer J. Influence of vasospasm on visual function. Doc Ophthalmol 1987;66:3-18.

6 Kitazawa Y, Shirai $\mathrm{H}$, Go FJ. The effect of $\mathrm{Ca}^{2+}$ antagonist on visual field in low tension glaucoma. Graefes Arch Clin Exp Ophthalmol 1989;227:408-12.

7 Netland PA, Chaturvedi N, Dreyer EB. Calcium channel blockers in the management of low tension and open angle glaucoma. Am f Ophthalmol 1993;115: 608-13.

8 Bose S, Piltz JR, Breton ME. Nimodipine, a centrally active calcium antagonist exerts a beneficial effect on contras sensitivity in patients with normal tension glaucoma and in control subjects. Ophthalmology 1995;102:1236-41.

Lieb WE, Cohen SM, Merton DA, Shields JA, Mitchell DG, Goldberg BB. Color Doppler imaging of the eye and orbit: technique and normal vascular anatomy. Arch Ophthalmol 1991;109:527-31.

10 Evans D, Harris A, Cantor L, Meyer M, Wilson R, Pillunat $\mathrm{L}$, et al. Failure of oral nifedipine to alter retrobulbar hemodynamics in normal tension glaucoma. Invest Ophthalmol Vis Sci 1995;36:S437.

11 Netland PA, Grosskreutz CL, Feke GT, Hart LJ. Color Doppler ultrasound analysis of ocular circulation after topical calcium channel blocker. Am $¥$ Ophthalmol 1995; 119:694-700.

12 Harris A, Williamson TH, Martin B, Shoemaker JA, Sergott RC, Speath HL, et al. Test/retest reproducibility of color Doppler imaging assessment of blood flow velocity in orbital vessels. F Glaucoma 1995;4:281-6. 